\title{
Transformaciones comunicativas en el ambiente de aprendizaje de una institución beneficiaria de CPE cuando los docentes desarrollan competencias técnicas y tecnológicas e incorporan TIC en la actividad conjunta
}

\author{
Communicative changes in the learning environment of an institution \\ beneficiary of CPE When Teachers develop technical and technological skills \\ and incorporate ICT in the Joint Activity
}

\author{
José Francisco Amador, Jorge Luis Rojas García ${ }^{2}$, Héctor Gerardo Sánchez Bedoya, Eduardo Duque \\ Amador Montaño, \\ 1, 2, ${ }^{3}$ Facultad de Ingenierías, Universidad Tecnológica de Pereira, Pereira, Colombia
}

jamador@utp.edu.co

\begin{abstract}
Resumen - Los ambientes de aprendizaje mediados por TIC son un espacio en el que se llevan a cabo procesos educativos donde la comunicación educativa tiene incidencia en las formas de enseñar y de aprender. En esta investigación se presenten determinar qué relaciones comunicativas de transforman en el interior de los ambientes de aprendizaje cuando los docentes incorporan TIC a sus prácticas educativas. Como conclusión principal en esta investigación se encuentran transformaciones comunicativas que van de lo análogo a lo digital, de lo privado a lo público, de lo autónomo a lo colaborativo, del reconocimiento de las oportunidades educativas que ofrece un entorno cada vez más mediatizado y digitalizado, para que los docentes que desarrollan competencias técnicas y tecnológicas produzcan $y$ propongan nuevas formas de representar y presentar la información para construir conocimiento de manera conjunta con los estudiantes en un ambiente de aprendizaje en el que las TIC se convierten en un apoyo de los procesos educativos y formativos.
\end{abstract}

Palabras clave-Comunicación educativa, ambiente de aprendizaje, aprendizaje mediado, aprendizaje, incorporación de TIC, actividad conjunta.

Abstract - Learning environments mediated by ICT is an area in which educational processes take place where the educational communication has an impact on the ways of teaching and learning. In this research are presented to determine what communicative relationships within transformed learning environments when teachers incorporate ICT into their teaching practices. The main conclusion in this investigation we found communicative transformations from the analogic to digital, from the private to public, from the self to collaborative, from recognition of the educative opportunities that give an environment each time more mediatized and digitalized, for the teachers who develop technical and technology competences they must produce and propose new ways to represent and show the information to build the knowledge together with their students in a environment learning where the ICT become in a support of the educative and formative process.

Key Word - Educational communication, learning environment, mediated learning, learning, incorporating ICT, joint activity.

\section{INTRODUCCIÓN}

Los docentes que se forman a través de la propuesta educativa de CPE disponen de un lenguaje y de recursos didácticos para la utilización de las TIC en el aula de clase. Con este conocimiento enfrentan situaciones anteriores a la realización de la clase que les determinan cambios en sus estrategias didácticas. De otro lado la misma forma de preparación de clase cuenta con soportes que le apoyan sus reflexiones que van a delimitar sus acciones en la clase cuando haga uso del computador o de servicios de red. Estas circunstancias llevan a que al interactuar con sus estudiantes y ellos con el computador las relaciones comunicativas tengan diferencias significativas frente a situaciones donde no hay uso de TIC en el aula. Esta investigación es pertinente porque posibilita evidenciar aquellos cambios que debe tener en cuenta un docente en sus actos comunicativos, por cuanto los tiempos se transforman, las formas de participar, las formas de representar están enmarcadas en otro escenario donde hay recursos para hacerlo y ya no es el docente la única fuente de información y de validación de la misma en el aula de clase.

\section{CONTENIDO}

\section{A. Justificación}

Los docentes que se forman a través de la propuesta educativa de CPE disponen de un lenguaje y de recursos 
didácticos para la utilización de las TIC en el aula de clase. Con este conocimiento enfrentan situaciones anteriores a la realización de la clase que les determinan cambios en sus estrategias didácticas. De otro lado la misma forma de preparación de clase cuenta con soportes que le apoyan sus reflexiones que van a delimitar sus acciones en la clase cuando haga uso del computador o de servicios de red. Estas circunstancias llevan a que al interactuar con sus estudiantes y ellos con el computador las relaciones comunicativas tengan diferencias significativas frente a situaciones donde no hay uso de TIC en el aula. Esta investigación es pertinente porque posibilita evidenciar aquellos cambios que debe tener en cuenta un docentes en sus acto comunicativo, por cuanto los tiempos se transforman, las formas de participar, las formas de representar están enmarcadas en otro escenario donde hay recursos para hacerlo y ya no es el docente la única fuente de información y de validación de la misma en el aula de clase.

\section{B. Pregunta de investigación}

¿Qué transformaciones comunicativas se presentan en un ambiente de aprendizaje de una institución beneficiaria del contrato CPE - UTP 2012 - 2014 cuando los docentes desarrollan competencias técnicas y tecnológicas e incorporan TIC en la actividad conjunta?

\section{Objetivo general}

Determinar las transformaciones comunicativas que se dan en un ambiente de aprendizaje de una institución beneficiaria del contrato CPE - UTP 2012 - 2014 cuando los docentes desarrollan competencias técnicas y tecnológicas e incorporan TIC en la actividad conjunta

\section{Objetivos específicos}

Identificar las competencias técnicas y tecnológicas que los docentes participantes en el proyecto de CPE desarrollan en su proceso de formación y llevan a sus ambientes de aprendizaje para la realización de su actividad docente.

Determinar el sistema de significados compartidos que se genera en los procesos de formación de los docentes participantes en el proyecto de CPE y aplican en sus ambientes de aprendizaje mediante los apoyos educativos que crean para sus clases.

Reconocer los procesos comunicativos que se dan en el ambiente de aprendizaje cuando se incorporan TIC como apoyo educativo.

\section{Marco teórico}

\section{Competencias técnicas y tecnológicas}

El desarrollo de competencias técnicas y tecnológicas por parte de los docentes y de las mismas instituciones que como ambientes de aprendizaje deben adaptarse y adoptar estrategias que posibiliten el ejercicio de docentes que tienen conocimiento acerca de la incorporación de las TIC en sus procesos de enseñanza y de aprendizaje.

\section{Desde CPE (Computadores para Educar)}

Atendiendo los conceptos derivados de las definiciones que se suscriben en el documento de los PLIEGOS DE CONDICIONES de la LICITACIÓN PÚBLICA No. 002 DE 2012 de CPE [11], se adoptará éstas en el tratamiento de la información que se procese y emane en esta investigación. Así, se transcribe de tal documento.

"Estándares de Competencias en la formación docente en TIC de Computadores para Educar

Competencias técnicas y tecnológicas

Son aquellas que hacen parte a una apropiación de las TIC, manejo y destrezas para navegar, apropiarse de las WEB 2.0 y 3.0 defenderse en un mundo tecnológico, y aprovecharlas para la vida misma y contribuir a la calidad educativa. Dimensionado sus potencialidades en el ámbito pedagógico."

Teniendo en cuenta que el proceso de formación docente se divide en 6 niveles que son la estructura del diplomado que cada docente hace en el transcurso de la intervención que hacen los gestores de CPE a través de la entidad operadora que para nuestro caso es la Universidad Tecnológica de Pereira.

\section{Desde el Ministerio de Educación}

Atendiendo las orientaciones emanadas desde la guía No 30 [20] del Ministerio de educación nacional, se discriminará y analizará el concepto de artefacto y de proceso así como de las relaciones que hay entre la tecnología y la técnica, la ciencia, la innovación, el diseño, la informática y la ética; como agentes directos de las transformaciones comunicativas en el proceso de enseñanza y de aprendizaje.

4. Representación visual del conocimiento y teorías del aprendizaje

Generalmente las representaciones visuales se encuentran combinadas con otros sistemas semióticos, especialmente la escritura, están constituidas principalmente por elementos gráficos, símbolos e imágenes que expresan relaciones funcionales y estructurales entre las distintas entidades representadas con un cierto nivel de abstracción.

La construcción de representaciones visuales afectan los procesos de enseñanza y aprendizaje debido a las posibilidades que ofrece, no solo para hacer públicos los significados construidos por los alumnos sobre los contenidos de aprendizaje, sino también para contrastarlos y negociarlos con el profesor y con otros alumnos. Los sistemas de representación visual son instrumentos psicológicos en el sentido Vygostkiano [16]; actúan como mediadores tanto de los procesos individuales (intramentales) del aprendiz, como de los procesos comunicativos y sociales (intermentales) implicados en el aprendizaje intencional. Esta función mediadora, no obstante, dependerá en buena medida de las características específicas de cada sistema de representación [26], es decir, de las restricciones 
derivadas de su propia y singular manera de representar la información.

\section{Aprendizaje colaborativo}

Según [4], el aprendizaje colaborativo hace referencia a "una actividad estructurada de aprendizaje que aborda las principales preocupaciones relacionadas con la mejora en la forma de aprender de los alumnos. Implica activamente a los estudiantes y los ayuda a valorar la perspectiva que cada uno puede aportar desde su experiencia personal académica... en otras palabras, el aprendizaje colaborativo es un método apropiado para conseguir algunos objetivos y realizar ciertas tareas" . La ventaja de una organización colaborativa del aprendizaje según Coll y Colomina (citados por [25]), radica en que "los objetivos que persiguen los participantes en las situaciones de aprendizaje, están estrechamente vinculadas entre sí, de tal manera que cada uno de ellos puede alcanzar sus objetivos, solo sí, los otros alcanzan los suyos" , por lo tanto, el aprendizaje colaborativo, según [25]" promueve los aprendizajes sociales (habilidades sociales, actitudes, etc.), así como se hace más posible la aparición de una motivación intrínseca por el aprendizaje o deseo de aprender, contribuyendo de esta manera a que se alcance un nivel de aprendizaje, o en su caso, un mejor rendimiento académico"

\section{Aprendizaje por indagación}

Tomado de [13] "se ha denominado pedagogía fundada o centradas en la indagación a las concepciones que colocan sus énfasis en: el problema, los dilemas, el método, la pregunta, la estrategia pedagógica, el lenguaje científico, el lenguaje cotidiano de los niños, niñas y jóvenes, los procedimientos. Desde estas diferentes posiciones, se considera que ello le debe permitir a los niños y niñas lograr un conocimiento propio de los contenidos escolares, así como un horizonte para usarlos en la vida ciudadana y cotidiana, a la vez que buscan un mundo donde se piensa, se actúa y se viva de una manera mejor.

El postulado: "Dímelo y se me olvidará, muéstramelo y lo recordaré, involúcrame y entenderé" es la esencia del aprendizaje por indagación.

\section{Enfoque pedagógico socioconstructivista}

Se conoce también como constructivismo de orientación sociocultural. Surge del intento por articular los planteamientos socioculturales y lingüísticos inspirados por Vigotsky y otros, con el constructivismo cognitvo de Piaget. El Socioconstructivismo concede especial importancia a los factores sociales; de acuerdo con Coll, Mauri y Onrubia (2007), sitúa la construcción del conocimiento en la actividad mental constructiva del alumno (procesos intrapsicológicos, inseparable de la actividad conjunta que desarrollan profesores y estudiantes en el contexto en que interactúan y de los procesos interpsicológicos que allí tienen lugar. Desde esta postura, la construcción del conocimiento en situaciones de enseñanza y aprendizaje es un proceso complejo de relaciones que se establecen entre los tres elementos del triángulo interactivo: el alumno que aprende, desarrollando su actividad mental de carácter constructivo; el contenido objeto de enseñanza y aprendizaje, y el profesor que ayuda al alumno en dicho proceso de construcción, progresando en el grado de significado sobre lo que aprende y siendo progresivamente más capaz de dotarle de sentido. El papel del profesor es el de orientar, guiar y sostener la actividad constructiva del alumno proporcionándole las ayudas educativas necesarias; mediando entre los saberes o contenidos de aprendizaje y la actividad del alumno. Los contenidos, por su parte, mediatizan la actividad conjunta de profesores y alumnos en el proceso de construcción. (Revista de Educación a Distancia. Teresa Mauri, Javier Onrubia, César Coll Rosa Colomina.)

\section{Incorporación de las TIC en el currículo}

Hablar de la incorporación de las TIC al currículo escolar significa tener y definir un enfoque pedagógico que le da direccionalidad y sentido. Al respecto se han formulado propuestas desde diferentes corrientes. Para el presente trabajo se ha asumido una concepción constructivista de la enseñanza y el aprendizaje escolar que pretende ofrecer un marco global de referencia psicológico que guíe y oriente el estudio de los procesos educativos, así como los esfuerzos dirigidos a una revisión y mejora. Este marco se inspira en la visión del constructivismo de orientación socio-cultural, surgido del intento de articular los planteamientos socio-culturales y lingüísticos, inspirados, entre otros, por la obra de Vigostky y sus continuadores, con el constructuvismo cognitivo, inspirado inicialmente en la obra de Piaget. Desde esta mirada, se sitúa en la actividad mental constructiva de los alumnos, y por tanto en la dinámica interna de los procesos intrapsicológicos de construcción del conocimientos, la clave del aprendizaje escolar; pero entiende al mismo tiempo, que esta dinámica interna se insiere necesariamente en, y resulta inseparable de, la actividad conjunta que desarrollan profesores y alumnos en el contexto particular en que interactúan.

\section{Las AHD (Ayudas Hipermediales Dinámicas)}

Una ayuda hipermedial dinámica (AHD) es un producto multimedia provisto de un sistema de hipertextualidad que lo dota de navegabilidad, de una estructura abierta que le permite transformarse y complementarse, de un conjunto de estrategias comunicativas, de un contenido específico, de un sistema de evaluación y de soporte específico para su manejo y uso en procesos de enseñanza y de aprendizaje desde un enfoque socioconstructivista.

\section{Convergencia digital}

Las transformaciones que se observan en la escuela tienen que ven con el paso de lo análogo a lo digital en aspectos que van desde el uso de medios públicos para comunicarse y expresar lo personal, hasta hacer uso de ellos para aspectos colaborativos en pro de la construcción del conocimiento. Estos cambios van de la 
mano con la evolución de ser lectores a ser productores de textos y de contenidos. A ejercer la ciudadanía digital y la exigencia de derechos como los de autos en un mundo cada vez más complejo en relaciones sociales a través de servicios y sistemas de vuelven invisible la línea que divide lo privado de lo público. Las maneras de expresión y de participación también hacen parte de este intrincado vínculo de esta convergencia digital. El proceso educativo no está sustraído de este dilema, al contrario, está en el centro de atención, dada cuenta que los estudiantes son usuarios intensivos de estas tecnologías de la información y la comunicación en espacios cada vez más abiertos y públicos [3].

\section{E. Marco Metodológico}

\section{Institución educativa}

Por recomendación de la dirección pedagógica de CPE, en esta investigación se incluye la comunidad educativa, esto es: estudiantes, profesores, padres de familia, directivos y administrativos.

El colegio que se ha seleccionado es la INSTITUCION EDUCATIVA INSTITUTO KENNEDY, ubicada en la ciudad de Pereira en el barrio Kennedy. Cuenda con dos sedes y atiende alrededor de 1900 estudiantes distribuidos entre las cuatro jornadas, para quienes se busca ofrecer una acción educativa que fortalezca su formación personal, en todas las dimensiones del ser humano y que beneficie la construcción de su proyecto de vida.

\section{CPE y la Institución educativa}

La institución educativa ha sido beneficiaria del programa CPE en años anteriores y en este contrato también. En la actualidad cuenta con dos salas de cómputo con equipos de escritorio, portátiles (70) y tabletas (30). Algunos de los docentes han sido formados por este proyecto.

La institución tiene articulación educación media con el SENA en programas técnicos de sistemas (data del año 2012) y diseño e integración de multimedios (desde el año 2013). Este programa de integración se lleva a cabo en las aulas con los equipos de cómputo existentes y con recursos adicionales del SENA. También cuenta con una sala de redes y mantenimiento de computadores donde los estudiantes de los dos programas de articulación hacen uso de ellas para realizar sus prácticas.

En esta relación con CPE se han formado aproximadamente 30 docentes de distintas áreas y niveles escolares, además, se han formado padres de familia que a su vez son estudiantes en las jornadas adicionales de educación de adultos.

Para la aplicación del instrumento de investigación se eligieron estudiantes (5), docentes (2), directivos (2) y padres de familia (2) de la comunidad académica.

El desarrollo de AHDs es el centro de los proyectos realizados en estas formaciones dada las características del proyecto ofrecido por la Universidad Tecnológica de Pereira como entidad operadora.
3. Integración de CPE con la Licenciatura en Comunicación e Informática Educativa de la Universidad Tecnológica de Pereira

La universidad Tecnológica de Pereira tiene dentro de sus programas de pregrado la Licenciatura en Comunicación e Informática Educativa, donde se formar docentes con un perfil orientado al uso de las TIC en procesos educativos. Dentro del currículo, los estudiantes adquieren conocimientos acerca de ambientes de aprendizaje y la intermediación de herramientas y mediaciones comunicativas que magnifican el proceso educativo. Como parte fundamental en esta carrera los estudiantes de últimos semestres desarrollan prácticas docentes y una de ellas es la que se integró al currículo llamada Práctica $\mathrm{CPE}$, en la cual los estudiantes aprenden sobre el proyecto, las dimensiones y su importancia en el contexto nacional. Son formados en el proyecto pedagógico propuesto por la UTP con el fin de que estén en contexto con el desarrollo de las actividades con las instituciones beneficiarias. Luego de esta fase acompañan a los gestores en las instituciones de la ciudad de Pereira, a quienes asisten en la aplicación de pruebas, la realización de actividades y aclaraciones sobre procedimientos y usos de herramientas informáticas en procesos educativos. También, apoyan los procesos con los padres de familia y colaboran con la optimización del material educativo que se entrega tanto a docentes como a padres de familia. Esta práctica docente está coordinada directamente por un profesor que orienta al equipo de estudiantes. Desde la dirección de pedagogía se lleva a cabo el proceso de formación y orientación para que se incorporen los practicantes al proceso. Se realiza un seguimiento par, debido a que tanto el docente de la Universidad como la dirección de pedagogía están pendientes del ejercicio y desarrollo de las actividades que se hacen. Por último, a los estudiantes - practicantes se le da una nota que es concertada con las actividades realizadas en este proyecto. Por último, muchos de los practicantes en esta modalidad deciden hacer su proyecto de grado, llamado Proyecto Pedagógico Mediatizado, en las instituciones beneficiarias de CPE, y se interesan por los temas que conciernen a los cambios y beneficios que obtienen las instituciones y la comunidad al disponer no solo de computadores sino de una formación que les permita generar transformaciones culturales y sociales en beneficio de ellas mismas.

\section{Tipo de investigación}

El alcance se esta investigación es descriptiva ya que busca especificar las características de las transformaciones comunicativas que ocurren en una institución educativa donde los docentes desarrollan competencias técnicas y tecnológicas. En este sentido vamos a indagar por esos cambios que se dan en la realidad cuando se hace uso de las TIC en la institución educativa.

El diseño de esta investigación es de corte cualitativo transeccional debido a que nos hay situaciones predefinidas, las variables corresponden a la situación real y actual. Atendiendo este diseño de investigación se aplicará un solo instrumento de investigación ya que solo se recopilarán datos en un momento único. Este momento 
es posterior a la finalización de la formación dada a los docentes y de la aplicación de los proyectos en Tic que llevaron a cabo con la asistencia de los gestores de CPE de la Universidad Tecnológica de Pereira. Desde la organización de este proceso, se aplica el instrumento de investigación terminado el nivel 6.

\section{Instrumento. Entrevista semiestructurada}

La entrevista es una forma comunicativa en la que dos personas intercambian información, teniendo en cuenta que el que tiene el rol de entrevistado es el que contesta las preguntas que el entrevistador hace de acuerdo a los objetivos de la investigación.

La entrevista semiestructurada tiene como principio informar acerca del propósito dela misma a quienes van a participar de ella. Por otro lado, en una entrevista de este tipo se hacen preguntas abiertas con el fin de obtener la mayor información posible acerca del tema que se esté tratando. Por último en estas entrevistas el guion no es explicito, ya que cada entrevistado puede presentar diferentes matices sobre una misma pregunta. De ahí, que la preguntas sean abiertas.

\section{Entrevistados}

Estudiantes: son las personas que están en proceso de aprendizaje. Para esta entrevista se tuvieron en cuenta diferentes grados de escolaridad (10 y 11) con el fin de recopilar la información desde distintos ángulos y percepciones desde el interior de las aulas, atendiendo que hagan un uso intensivo del computador.

Profesores: personas que ejercen la profesión docente en el colegio y que han tenido formación en el uso de las TIC a través del proyecto de CPE.

Directivos: son las personas encargadas de la organización de la institución desde lo académico hasta lo administrativo. Para la entrevista asistieron la rectora de la institución y un coordinador.

Padres de familia: son las personas que responden con acudientes de los estudiantes, se tuvo en cuenta a dos de ellos, uno cuyo hijo es de primaria y otro que tiene su hijo en media.

7. Análisis de la investigación (Procedimiento para el análisis de la información)

Atendiendo a los objetivos de la investigación se procederá a organizar la información recolectada a través de las transcripciones de las entrevistas. En primera instancia se ingresará en una tabla las menciones de los docentes que permiten ver el desarrollo de competencias técnica y tecnológicas a partir de los desempeños de los estándares en competencias establecidos por CPE desde lo pliego del proyecto CPE-UTP 2012-2014. De donde se interpretará acerca del uso de las TIC en la actividad docente a partir del proceso de formación de los docentes. Como segunda parte se tendrán en cuenta los significados compartidos entre docentes, estudiantes, directivos y padres de familia en lo atinente a las maneras de utilizar las TIC como apoyo educativo. Por último se tendrá en cuenta los procesos comunicativos en los grupos de personas entrevistadas relacionados con el concepto de convergencia digital, es decir el paso de lo análogo a la digital, sus usos, su importancia y el estado actual de estas transformaciones.

\section{CONCLUSIONES}

Esta investigación tiene como objetivo central el determinar las transformaciones comunicativas que se dan en un ambiente de aprendizaje de una institución beneficiaria del contrato CPE - UTP 2012 - 2014 cuando los docentes desarrollan competencias técnicas y tecnológicas e incorporan TIC en la actividad conjunta. Para lo cual hemos trazado un camino de tres objetivos que nos permitan evidenciar estar transformaciones comunicativas.

Atendiendo el objetivo de Identificar las competencias técnicas y tecnológicas que los docentes participantes en el proyecto de CPE desarrollan en su proceso de formación y llevan a sus ambientes de aprendizaje para la realización de su actividad docente, se encontró que los docentes han desarrollado competencias técnicas y tecnológicas de manera significativa para hacer parte de su hacer cotidiano en el interior del aula. Máxime cuando distingue situaciones problémicas y las enfrentan con propiedad y convicción con el fin de ofrecer al estudiante un espacio de acción correspondiente con la época en términos de recursos y dispositivos informáticos que están al alcance de los estudiantes. Esta es una evidencia importante de resalta en términos de las oportunidades en términos de la formación docente a la hora de implementas una AHD en medio de un DTP, por cuando esta propuesta visualiza al docente un actor activo en el uso de las TIC para adaptar y adoptar en procesos educativos de diversa condiciones. Por eso, la flexibilidad con la que los docentes enfrentan la realidad y hacen ajustes pertinentes desde lo técnico y lo tecnológico inciden en lo pedagógico y exhiben fortaleza en sus estudiantes quienes se comunican con ellos de manera connatural de los recursos y medios con los cuales interactúan para aprender de manera independiente y colaborativa.

Con respecto al objetivo de determinar el sistema de significados compartidos que se genera en los procesos de formación de los docentes participantes en el proyecto de CPE y aplican en sus ambientes de aprendizaje mediante los apoyos educativos que crean para sus clases, se encontró que entre los estudiantes hay un sistema de significados compartidos que está evolucionando y que se consolida a través de las prácticas que hacen a la hora de realizar actividades educativas. Este es un elemento importante dentro del aprendizaje autónomo y colaborativo, los cuales se tienen en cuenta en la formación que reciben los profesores a través de los gestores de CPE, cuando están eligiendo y valorando material digital para implementar en las AHD. De otra parte entre docentes se observó que también tienen significados compartidos cuando se comunican con sus estudiantes acerca de la manera como solicitan trabajos 
escritos y orientan las actividades mediante recursos de la web 2.0. Esto lo evidencian los mimos estudiantes quienes se sienten acompañados por sus docentes cuando están en contacto virtual mediante plataformas de redes sociales o del mismo correo electrónico. Con respecto a los directivos, expresan coherencia en términos de la utilidad organizaciones y logísticas para posibilitar las acciones tanto de docentes, estudiantes y padres de familia, Además, ofrecen un escenario de transición para que las personas se incluyan en estos procesos de digitalización de la información. Resulta también significativo que entre los padres de familia y los estudiantes hay un escaso nivel en la construcción de significados compartidos, dada cuenta las creencias manifestadas por los padres. Los estudiantes reconocen las limitaciones en estos aspectos de los padres y optan por ofrecer su disposición para enseñarles acerca del tema.

Con respecto al objetivo de reconocer los procesos comunicativos que se dan en el ambiente de aprendizaje cuando se incorporan TIC como apoyo educativo, teniendo en cuenta la convergencia digital en términos del paso de lo análogo a lo digital se encontró que en los procesos comunicativos entre estudiantes es connatural la manera de construir un diálogo con y acerca de recursos digitales, de plataforma comunicativas y de escenarios para recibir asistencia educativa. Son nativos digitales en términos de Piscitelli. Con respecto a los docentes se evidencia una formación que les posibilita entrar a comunicarse con los estudiantes y descubren en los recursos digitales posibilidades para enseñar y apara aprender, teniendo en cuenta que identifican producen material educativo tal como las AHD y tienen estrategias para su uso tal como un DTP. Con respecto a los directivos se evidencia disposición para crear estrategias y logísticas para que la comunicación fluya por medios digitales, para estudiantes, profesores y padres de familia.

Para finalizar, se observan transformaciones comunicativas que van de lo análogo a lo digital, de lo privado a lo público, de lo autónomo a lo colaborativo, del reconocimiento de las oportunidades educativas que ofrece un entorno cada vez más mediatizado y digitalizado, para que los docentes que desarrollan competencias técnicas y tecnológicas produzcan y propongan nuevas formas de representar y presentar la información para construir conocimiento de manera conjunta son los estudiantes en un ambiente de aprendizaje en el que las TIC se convierten en una apoyo de los procesos educativos y formativos.

\section{REFERENCIAS}

[1] Anderson, R. C. (1984). Some reflections on the acquisition of knowledge. . Educational Researcher, 5-10.

[2] Ausubel, D. P. (2002). Adquisición y retención del conocimiento. Barcelona: Paidós.

[3] Barbero, J. M. (2001). Transformacionescomunicativas y tecnológicasde lo público. Metapolítica, 46-55.
[4] Barkley, Elizabeth; Cross, K. Patricia \& Howell Major, Claire (2007). Técnicas de aprendizaje colaborativo. Madrid: Ministerio de Educación y Ciencia/Morata.

[5] César, C., Martín, E., Mauri, T., Miras, M., Onrubia, J., Solé, I., y otros. (1999). El constructuvismos en el aula. Barcelona: Graó.

[6] Coll, C., \& Martí, E. (s.f.). Recuperado el 2 de Agosto de 2012, de La educación escolar ante las nuevas tecnologías de la información y de la comunicación.:

[7] Coll, C., \& Monereo, C. (2008). Psicología de la educación virtual. Aprender y enseñar con las tecnologías de la información y la comunicación. Madrid: Morata.

[8] Coll, C., Colomina, R., Onrubia, J., \& Rochera, M. J. (1992). Actividad conjunta y habla: una aproximación al estudio de los mecanismos de influencia educativa. Infancia y aprendizaje, 59 -60, 189 - 232.

[9] Coll., C. (2004). Psicología de la educación y prácticas educativas mediadas por las tecnologías de la información y la comunicación. Una mirada constructivista. Sinéctica, 1-24.

[10] Colomina, R., \& Otros. (2001). Interactividad, mecanismos de influencia educativa y construcción del conocimiento en el aula. Desarrollo Psicológico y Educación 2. Psicología de la Educación, 437-458.

[11] CPE, A. C. (Mayo de 2012). PLIEGO DE CONDICIONES LICITACIÓN PÚBLICA No. 002 DE 2012. Bogotá D.C, Colombia.

[12] Erickson, F. (1982). Classroom Discourse as Improvisation: Relationships between academic Task Structure and Social Participation Structure in Lesson. Communicating in the classroom, 153-181.

[13] Escalante, P. (s.f.). APRENDIZAJE POR INDAGACIÓN. Recuperado el agosto de 2012, de http://www.medellin.edu.co/sites/Educativo/repositorio\% 20de\%20recursos/Aprendizaje\%20por\%20indagaci\%C3 \%B3n.pdf

[14] Glaser, R. y. (1989). Learning theory and the study of. Annual Review of Psychology,40, 631-666.

[15] Grané, M., \& Willen, C. (2009). Web 2.0: nuevas formas de aprender y de participar. Barcelona: Laertes S.A.

[16] Gros, B. (2008). Aprendizaje, conexiones y artefactos. La producción colaborativa del conocimiento. Barcelona: Gedisa.

[17] Gutiérrez, M. C., \& Buitrago, O. (2009). Interactividad e influencia educativa: la construcción de conocimiento en el programa ondas de colciencias con apoyo de tic (Memorias, I congreso Nacional de 
investigación en educación en ciencias y tecnología, 2009, Junio 22 a 26, ISBN: 978-958-99491-0-). Bogotá: Asociación Colombiana para la investigación en Ciencias Y Tecnología EDUCyT.

[18] Hernández, V. (2007). Mapas conceptuales. México D.F: Alfaomega.

[19] Landow, G. (2006). Hipertexto 3.0. Barcelona: Paidós.

[20] MEN. (mayo de 2008). Ser competente en tecnología. Recuperado el 21 de Enero de 2013, de http://www.mineducacion.gov.co/1621/articles-

160915_archivo_pdf.pdf

[21] Mononen-Aaltonen, M. (s.f.). A Learning Environment-A Euphemism for Instruction or a Potential for Dialogue? Recuperado el 2 de dic de 2012, de http://hrast.pef.unilj.si/ joze/podiplomci/FRI/mep8/Mononen-Aaltonen.pdf

[22] Novak, J. D. (1998). Learning How to Learn. Cambridge: Cambridge University Press.

[23] Onrubia, J. (2005). Aprender y enseñar en entornos virtuales: actividad conjunta, ayuda pedagógica y construcción del conocimient. Revista de Educación a Distancia., 1 - 16.

[24] Ontoria, A. (2006). Mapas conceptuales. Una técnica para aprender. Madrid: Narcea.

[25]Pozo. J, I (2005). Adquisición del conocimiento. Morata

[26] Rey, F. (Diciembre de 2008). Utilización de los mapas conceptuales como herramienta evaluadora del aprendizaje significativo del alumno universitario en ciencias con independencia de su conocimiento de

la metodología. Recuperado el 4 de Febrero de 2012, de Tesis doctoral: http://www.tdx.cat/bitstream/handle/10803/9267/Utilizaci on_de_los_mapas_conceptuales_como_herramienta_eval uadora_del_aprendizaje_significati.pdf?sequence $=1$

[27] Schnotz, W. (2002). Towards an integrated view of learning from text and visual displays. Educational Psychology Review, 101-120.

[28] Scolari, C. (2004). Hacer clic. Barcelona: Gedisa.

[29] UNESCO. (8 de Enero de 2008). ESTÁNDARES DE COMPETENCIAS EN TIC PARA DOCENTES. Recuperado el 3 de dic de 2012, de http://www.eduteka.org/pdfdir/UNESCOEstandaresDoce ntes.pdf. 